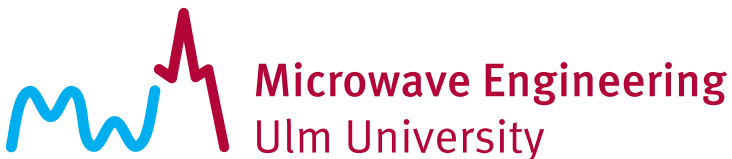

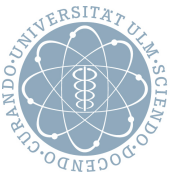

\section{Tripwire Detection in SAR Images Using a Modified Radon Transform}

Markus Schartel, Alexander Grathwohl, Christopher Schmid, Ralf Burr and Christian Waldschmidt

(C) 2020 IEEE. Personal use of this material is permitted. Permission from IEEE must be obtained for all other uses, in any current or future media, including reprinting/republishing this material for advertising or promotional purposes, creating new collective works, for resale or redistribution to servers or lists, or reuse of any copyrighted component of this work in other works. 


\title{
TRIPWIRE DETECTION IN SAR IMAGES USING A MODIFIED RADON TRANSFORM
}

\author{
Markus Schartel ${ }^{*}$, Alexander Grathwohl ${ }^{*}$, Christopher Schmid ${ }^{*}$, Ralf Burr $^{\dagger}$, \\ and Christian Waldschmidt* \\ *Institute of Microwave Engineering, Ulm University, 89081, Germany \\ †Ulm University of Applied Sciences, 89075 Ulm, Germany \\ Email: markus.schartel@alumni.uni-ulm.de
}

\begin{abstract}
Anti-personnel fragmentation mines and improvised explosive devices often use metallic wires-so-called tripwiresfor the trigger mechanism. Depending on environment and vegetation, these wires are hardly visible. Therefore, an airborne synthetic aperture radar (SAR) was developed to assist the process of mine clearance. In this work, an algorithm for the detection and localization of tripwires in SAR images is proposed. A modified Radon transform is employed that relies on the SAR phase information. Instead of simply integrating absolute values over a straight line, the complex-valued data is analyzed in the frequency domain. The result in the transformed image is then given by the maximum amplitude within a given frequency range, causing tripwires to clearly separate from clutter. The functionality of this algorithm is successfully demonstrated by measurements of tripwires attached to a dummy mine in wet grass.
\end{abstract}

Index Terms - anti-personnel mine, unmanned aerial system, multicopter, synthetic aperture radar, tripwire, radon transform

\section{INTRODUCTION}

In humanitarian demining operations, anti-personnel (AP) fragmentation mines pose a major threat to human lives. These mines are larger in comparison to blast mines, contain a high metal content, and can have a large lethal range of over $50 \mathrm{~m}[1]$. Usually, they are only partially buried and employ an area fuzing system using tripwires. The mine itself should be easy to identify visually or by using a metal detector. The challenge is the detection and localization of thin metal tripwires without triggering the mine from a distance. The same holds true for improvised explosive devices. Currently, the procedure of finding tripwires in mine clearing operations is carried out by hand, as metal detectors do not detect the wires. This process is very time consuming and highly dangerous. The use of an autonomous flying sensor platform able to detect tripwires would improve this process.

In [2], an unmanned aerial system (UAS) using a synthetic aperture radar (SAR) has been demonstrated, which can be

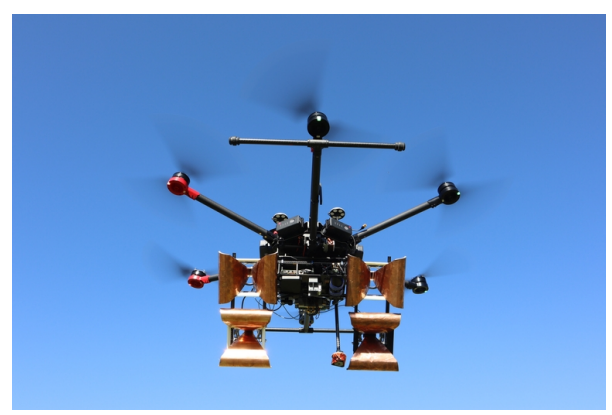

Fig. 1: UAS equipped with FMCW SAR and RTK GNSS.

used to detect vegetation-obscured tripwires. It was shown that thin metallic wires may be difficult to identify in SAR images, especially under wet conditions. Hence, an algorithm for the reliable automatic detection and localization of tripwires employing a modified Radon transform is proposed in this work.

This paper is structured as follows: The measurement system is presented briefly in Section 2. Section 3 presents the proposed detection algorithm. In Section 4, this algorithm is verified using SAR images of tripwires tensioned in dewy grass. Section 5 gives the conclusion.

\section{MEASUREMENT SYSTEM}

The measurement system is shown in Fig. 1. An industrial multicopter with an integrated inertial measurement unit [3] was equipped with a real time kinematic global navigation satellite system (RTK GNSS) [4] and a bistatic frequencymodulated continuous-wave (FMCW) SAR [5]. Due to the highly nonlinear flight path, a back-projection algorithm was used for the SAR image generation [2]. The radar was operated in a frequency range from $1 \mathrm{GHz}$ to $2.3 \mathrm{GHz}$ with a transmit power of $15 \mathrm{dBm}$. The maximum chirp repetition frequency $(\mathrm{CRF})$ is $30 \mathrm{~Hz}$. The horn antennas have a $3 \mathrm{~dB}$ beamwidth of approximately $60^{\circ}$ in the E-plane and $50^{\circ}$ in the H-plane. 


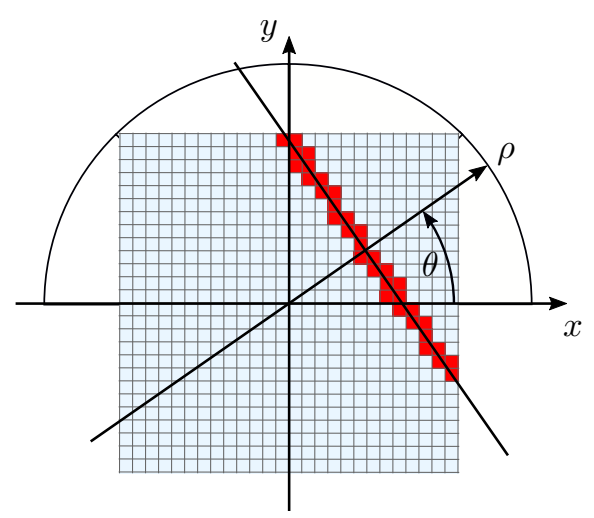

Fig. 2: Extraction of lines from the complex SAR image [8].

\section{TRIPWIRE DETECTION}

The Radon transform developed by Johann Radon in 1917 is an integral transformation suited to highlight and detect lines in images [6]. For a two-dimensional input image $A(x, y)$ it can be defined as [7], [8]:

$$
r(\theta, \rho)=\int_{-\infty}^{\infty} \int_{-\infty}^{\infty} A(x, y) \delta(\rho-x \cos \theta-y \sin \theta) \mathrm{d} x \mathrm{~d} y .
$$

$\theta \in[0,180)$ represents an angle in degrees and $\rho \in \mathbb{R}$ a distance from the origin. The Dirac function $\delta(\cdot)$ is used to select data from the input image on a line defined by the equation $\rho-x \cos \theta-y \sin \theta=0$, as illustrated in Fig. 2. In practice, $A(x, y)$ is not continuous, but consists of discrete pixels. Therefore, linear interpolation between neighboring pixels is used to compute a vector $\mathbf{v}$ of values for every line [7], [8]. In the original transformation the data on this line would be integrated to calculate $r(\theta, \rho)$. By integration signals with a line-like characteristic are concentrated into a single maximum in the transformed image. In the application of the original transform on SAR images, only the amplitude but not the phase is considered.

However, elongated objects cause a characteristic phase signature, which can be used to detect wires [9]. Fig. 3 shows a SAR image of a setup consisting of five tripwires and four corner reflectors, Fig. 4 a section of the phase of this SAR image. Tripwires cause an approximately constant phase in longitudinal direction, the phase progression in transversal direction is approximately periodic.

The phase along three lines with $\theta=50^{\circ}, 60^{\circ}$, and $70^{\circ}$ is shown in Fig. 5. If the extracted line is located on a tripwire and runs parallel to it, the phase progression over the line is almost constant $\left(\theta=60^{\circ}, \rho=-82\right.$ pixel). If there is a small deviation in $\theta$, the phase progression is linear $(\theta=$ $50^{\circ} / 70^{\circ}, \rho=-82$ pixel). In order to integrate the SAR phase into the detection and to increase reliability, the Radon transform is modified according to [9].

Fig. 6 visualizes the modified algorithm. Instead of ex-

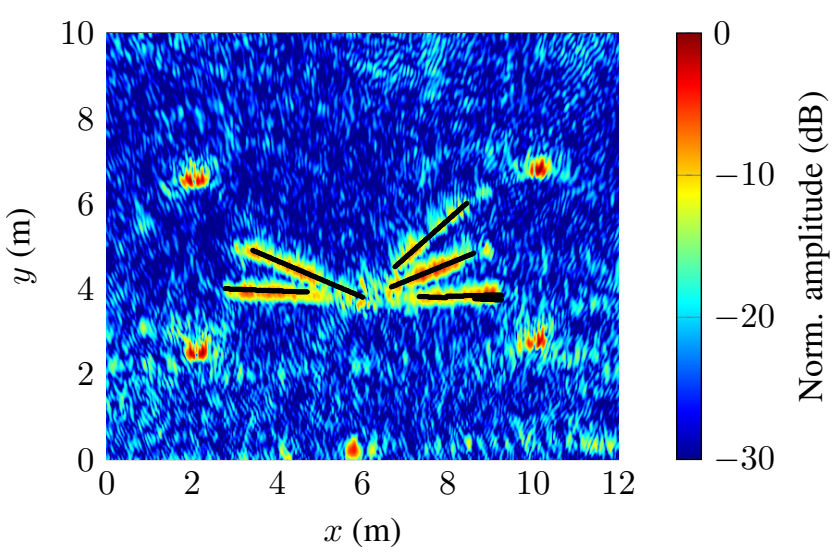

Fig. 3: SAR image, in which five tripwires and four corner reflectors are visible. The tripwires are detected and highlighted automatically by the algorithm.

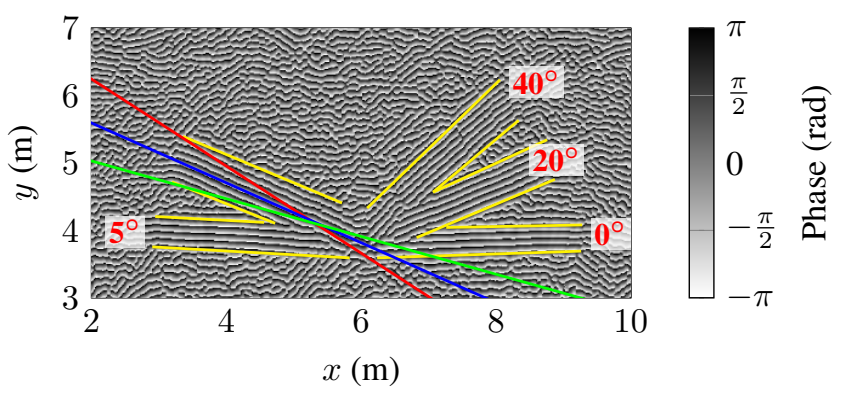

Fig. 4: Phase information of the SAR image shown in Fig. 3. Wires lead to a characteristic signature with constant phase.

tracting only the amplitude for every line, a complex-valued vector $\mathbf{v}$ of length $M$ is calculated. The areas of linear phase progression then correspond to a low-frequency component. This component can be extracted by applying a Hann-window and calculating the Fourier transform $\mathcal{F}\{\mathbf{v}[k]\}$ over each line.

First, the resulting spectrum is normalized. By choosing $k$, the maximum frequency $f_{g}=\frac{M}{k}$ still considered to be part of the signature of a tripwire is selected. Then, the maximum amplitude in the frequency range $\left[-f_{g}, f_{g}\right]$ is determined. This corresponds to a low-pass filter removing highfrequency noise and clutter. If the corresponding frequency is negative, the amplitude is multiplied by -1 . These processing steps are performed for all parameters $\theta$ and $\rho$.

The modified transform of the SAR image (Fig. 3) is shown in Fig. 7. The five wires are represented by five ovalshaped extrema. For the parameters $\theta$ and $\rho$ matching a tripwire, the phase progression is constant and the resulting frequency is zero. Deviations in $\theta$ result in a linear phase progression and therefore in a positive or negative frequency component. Hence, for every wire two local extrema with opposite sign are visible next to each other after the transformation. 

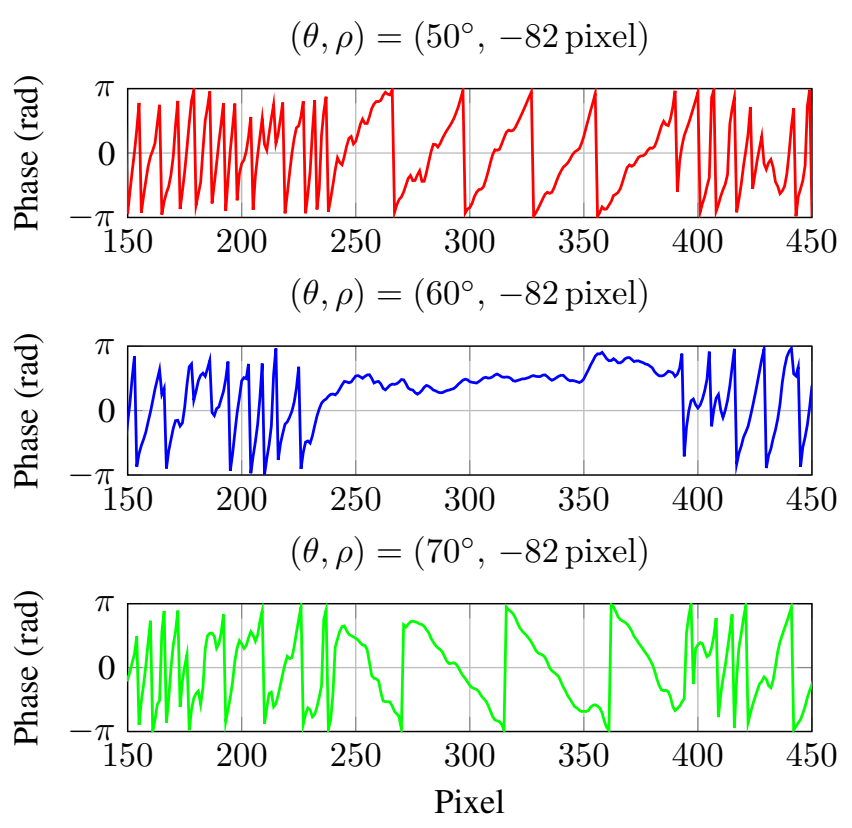

Fig. 5: Phase progression over extracted lines from Fig. 4.

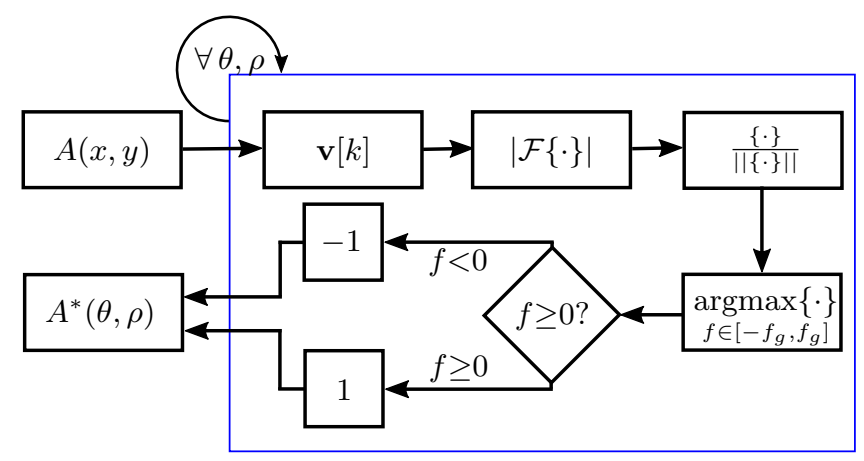

Fig. 6: Block diagram to illustrate the modified Radon transform.

Now, a Sobel filter is used to find the zero crossings between these extrema. Then, a threshold is applied to distinguish between tripwires and clutter as shown in Fig. 8. The parameters for straight lines, which tripwires are located on, can be extracted by a maximum search for every cluster in Fig. 8. The corresponding lines can then be displayed in the SAR image (see Fig. 9).

Although tripwires are located on the previously generated lines, their position and length are still unknown. To determine their exact location, the vectors $\mathbf{v}$ for the corresponding lines are split into blocks of equal length. After applying a Hann-window and the Fourier transform, every block is again analyzed in the frequency domain. Since the tripwires are already aligned, they cause a DC-component in the resulting spectrum (see Fig. 10). By applying a threshold, the blocks containing a tripwire signature, namely a constant phase, can

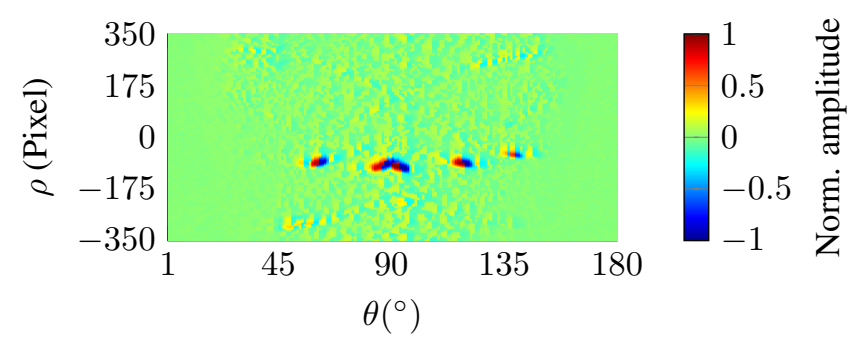

Fig. 7: SAR image after applying the modified Radon transform: $A^{*}(\theta, \rho)$.

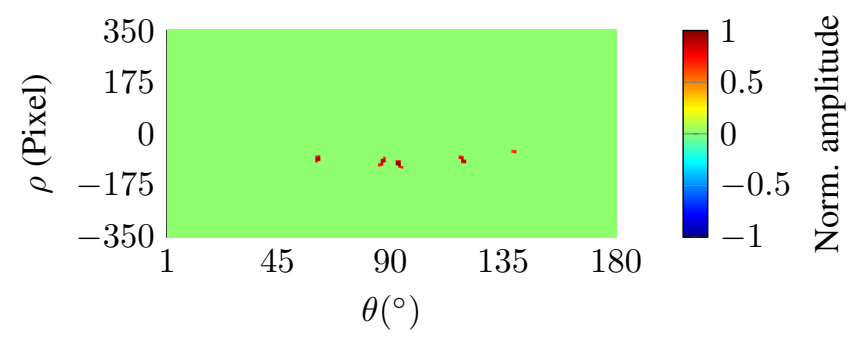

Fig. 8: $A^{*}(\theta, \rho)$ after applying a Sobel-filter and a threshold.

be extracted. Finally, these areas of the lines can be highlighted in the original SAR image, as shown in Fig. 3.

\section{MEASUREMENT RESULTS}

To recreate a realistic scenario, four wires with a diameter of $0.5 \mathrm{~mm}$ and a length of $3 \mathrm{~m}$ were connected to a dummy mine and tensioned at a height of about $15 \mathrm{~cm}$ over the ground. Additionally, four corner reflectors were placed as a reference [2]. For this setup a stripmap SAR measurement was taken at a height of approximately $3.5 \mathrm{~m}$ with a length of around $30 \mathrm{~m}$. The measurement took place early in the morning, so the grass was still wet with dew. As a result, the wires in the SAR image shown in Fig. 12 are hardly visible. Nonetheless, all four tripwires are detectable and locatable by

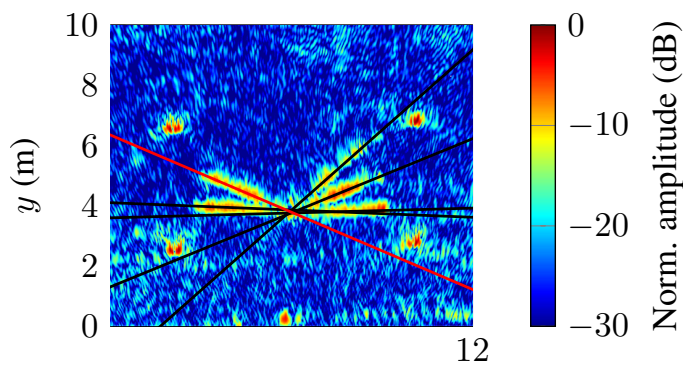

$x(\mathrm{~m})$

Fig. 9: Lines (- and - - ) on which tripwires are detected, plotted in SAR image. 


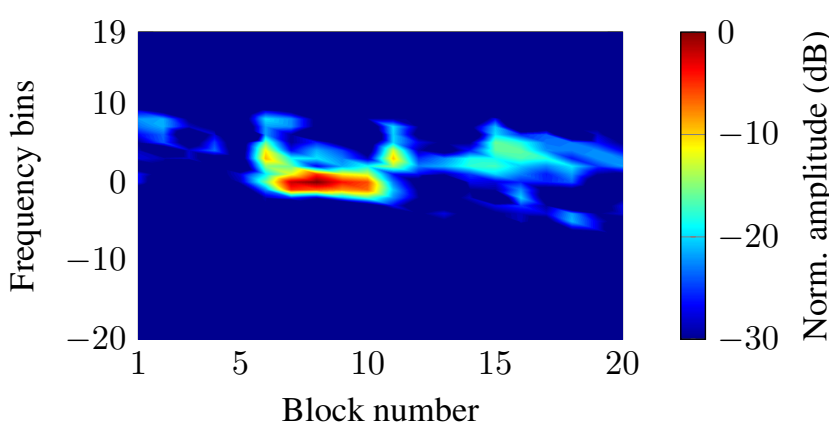

Fig. 10: Block-wise Fourier transform over vector $\mathbf{v}$ for line $(-)$ in Fig. 9.

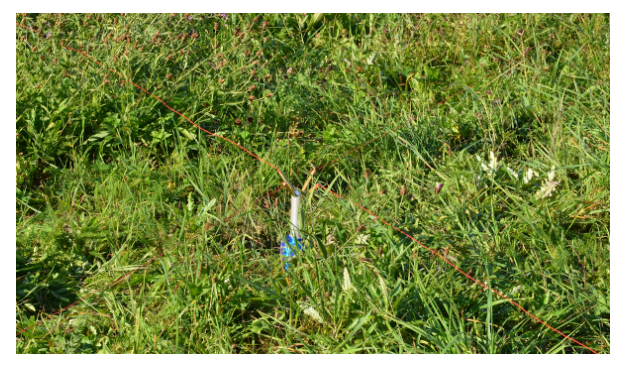

Fig. 11: Photo of a dummy mine with four tensioned wires with a diameter of $0.5 \mathrm{~mm}$.

the proposed method, as is visible in Fig. 13. The line for one of the detected wires crosses the high signal amplitude of a corner reflector. Therefore, the detected tripwire extends beyond the reflector location.

\section{CONCLUSION}

In this work, an algorithm for the detection and localization of tripwires in SAR images was proposed. The Radon transform was modified to include the phase information of the SAR images. In contrast to the Radon transform, where the amplitude on a straight line is integrated, the key element in this

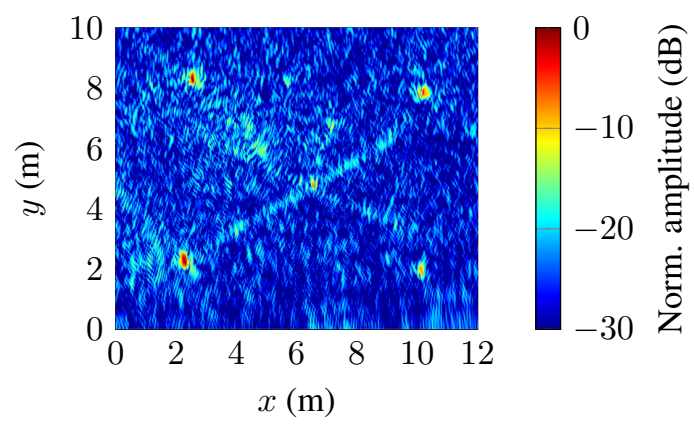

Fig. 12: SAR image of the measurement setup with four tripwires and four corner reflectors.

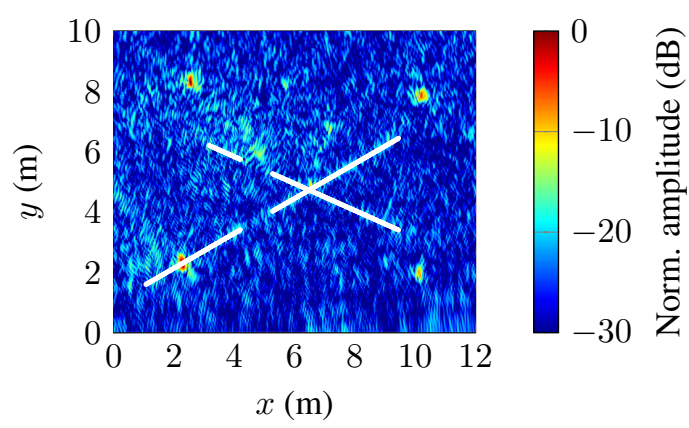

Fig. 13: SAR image of the measurement setup with four tripwires and four corner reflectors, resulting detected tripwires are highlighted.

transform is an analysis of frequency components on these lines. It was shown, that tensioned tripwires with a diameter of $0.5 \mathrm{~mm}$ can be detected close to the ground in an environment with wet grass, despite of being hardly visible in the SAR image.

\section{REFERENCES}

[1] C. King, "Demining: enhancing the process," in Second International Conference on the Detection of Abandoned Land Mines, Oct. 1998.

[2] M. Schartel, R. Burr, W. Mayer, and C. Waldschmidt, "Airborne Tripwire Detection Using a Synthetic Aperture Radar," IEEE Geoscience and Remote Sensing Letters, June 2019.

[3] DJI, Matrice 600 Pro User Manual, 2018, V1.0 2018.04.

[4] DJI, “D-RTK Release Notes,” 2017.

[5] R. Burr, M. Schartel, P. Schmidt, W. Mayer, T. Walter, and C. Waldschmidt, "Design and Implementation of a FMCW GPR for UAV-based Mine Detection," in IEEE MTT-S International Conference on Microwaves for Intelligent Mobility (ICMIM), 2018.

[6] J. Radon, "On the Determination of Functions From Their Integral Values Along Certain Manifolds," IEEE Transactions on Medical Imaging, vol. 5, no. 4, pp. 170176, Dec 1986.

[7] Peter Aundal Toft and John Aasted Sørensen, The Radon Transform - Theory and Implementation, Ph.D. thesis, Technical University of Denmark (DTU), 1996.

[8] Carsten Høilund, “The Radon Transform," Aalborg University, 2007.

[9] J. K. Jao and S. Ayasli, "SAR Detection Of Wires Using Image Phase Signatures," in Proceedings International Radar Conference, May 1995, pp. 362-368. 